\title{
Thyroid hormone synthesis and anti-thyroid drugs: A bioinorganic chemistry approach
}

\author{
GOURIPRASANNA ROY and G MUGESH* \\ Department of Inorganic \& Physical Chemistry, Indian Institute of Science, Bangalore 560012 \\ e-mail: mugesh@ipc.iisc.ernet.in
}

\begin{abstract}
Hydrogen peroxide, generated by thyroid oxidase enzymes, is a crucial substrate for the thyroid peroxidase (TPO)-catalysed biosynthesis of thyroid hormones, thyroxine (T4) and triiodothyronine (T3) in the thyroid gland. It is believed that the $\mathrm{H}_{2} \mathrm{O}_{2}$ generation is a limiting step in thyroid hormone synthesis. Therefore, the control of hydrogen peroxide concentration is one of the possible mechanisms for the inhibition of thyroid hormone biosynthesis. The inhibition of thyroid hormone synthesis is required for the treatment of hyperthyroidism and this can be achieved by one or more anti-thyroid drugs. The most widely used anti-thyroid drug methimazole (MMI) inhibits the production of thyroid hormones by irreversibly inactivating the enzyme TPO. Our studies show that the replacement of sulphur in MMI by selenium leads to a selone, which exists predominantly in its zwitterionic form. In contrast to the sulphur drug, the selenium analogue (MSeI) reversibly inhibits the peroxidase-catalysed oxidation and iodination reactions. Theoretical studies on MSeI reveal that the selenium atom in this compound carries a large negative charge. The carbon-selenium bond length in MSeI is found to be close to single-bond length. As the selenium atom exhibits a large nucleophilic character, the selenium analogue of MMI may scavenge the hydrogen peroxide present in the thyroid cells, which may lead to a reversible inhibition of thyroid hormone biosynthesis.
\end{abstract}

Keywords. Antioxidants; anti-thyroid drugs; iodination; selenium; thyroxine.

\section{Introduction}

Thyroxine (T4), the main secretory hormone of the thyroid gland, is produced on thyroglobulin by thyroid peroxidase (TPO)/hydrogen peroxide/iodide system. The synthesis of T4 by TPO involves two independent steps: iodination of tyrosine and phenolic coupling of the resulting iodotyrosine residues (figure 1). ${ }^{1}$ The prohormone $\mathbf{T 4}$ is then converted to its biologically active form $\mathbf{T 3}$ by iodothyronine deiodinase (ID-I), which is present in highest amounts in liver, kidney, thyroid and pituitary. ${ }^{2}$ Although the deiodination reactions are essential for the function of the thyroid gland, the activation of thyroid stimulating hormone (TSH) receptor by auto-antibodies leads to an overproduction of thyroid hormones. As these antibodies are not under pituitary feedback control system, there is no negative influence on the thyroid activity and, therefore, the uncontrolled production of thyroid hormones leads to a condition called "hyperthyroidism" (figure 2). Under these conditions, the overproduction of $\mathbf{T 4}$ and $\mathbf{T 3}$ can be controlled by specific inhibitors, which either block

\footnotetext{
*For correspondence
}

the thyroid hormone biosynthesis or reduce the conversion of $\mathbf{T} 4$ to $\mathbf{T 3}$. A unique class of such inhibitors are the thiourea drugs, methimazole (1, MMI), 6-n-propyl-2-thiouracil (3, PTU), and 6-methyl-2thiouracil (5, MTU) (figure 2).

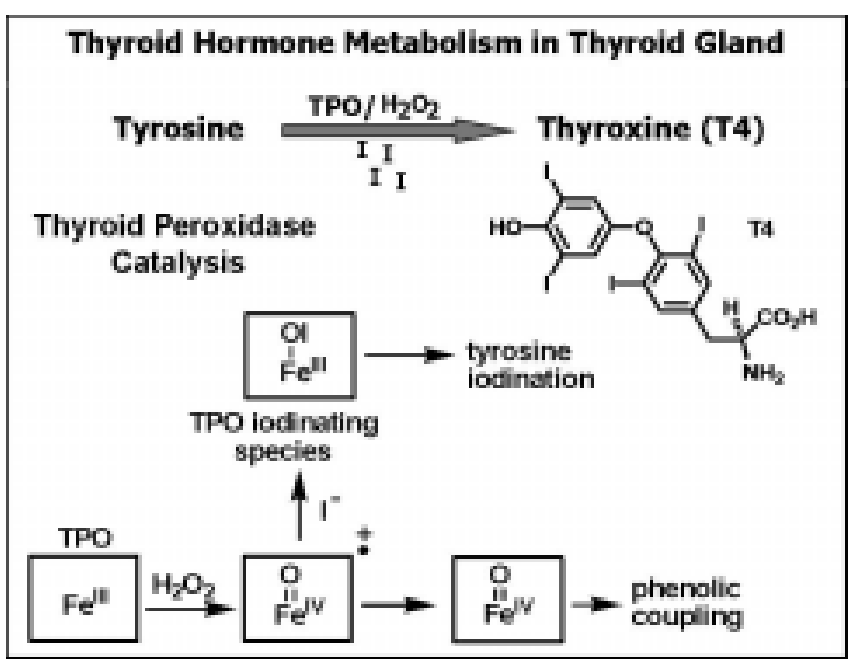

Figure 1. Synthesis of thyroid hormones by hemecontaining thyroid peroxidase (TPO). 
Although these compounds are the most commonly employed drugs in the treatment of hyperthyroidism, the detailed mechanism of their action is still not clear. According to the initially proposed mechanism, these drugs may divert oxidized iodides away from thyroglobulin by forming stable electron donoracceptor complexes with diiodine, which can effectively reduce thyroid hormone biosynthesis. ${ }^{3}$ It has also been proposed that these drugs may block the thyroid hormone synthesis by coordinating to the metal centre of thyroid peroxidase (TPO). ${ }^{4}$ After the discovery that ID-I is responsible for the activation of thyroxine, it has been reported that PTU, but not MMI, reacts with the selenenyl iodide intermediate (E-SeI) of ID-I to form a selenenyl sulphide as a dead end product, thereby blocking the conversion of T4 to T3 during the monodeiodination reaction. ${ }^{2}$ The mechanism of anti-thyroid activity is further complicated by the fact that gold-containing drugs such as gold thioglucose (GTG) inhibit the deiodinase activity by reacting with the selenol group of the native enzyme. ${ }^{2}$

Recently, the selenium analogues 2 (MSeI), 4 (PSeU) and 6 (MSeU) attracted considerable attention. ${ }^{5,6}$ However, the data derived from the inhibition of TPO by selenium compounds show that these compounds may inhibit the TPO activity by a different mechanism. Therefore, further studies are required to understand the mechanism by which the selenium compounds exert their inhibitory action. Our initial attempts to isolate $\mathbf{2}$ were unsuccessful and the final stable compound in the synthesis was characterized to be the diselenide $(\mathbf{8})$. In view of the current interest in anti-thyroid drugs and their mechanism, we

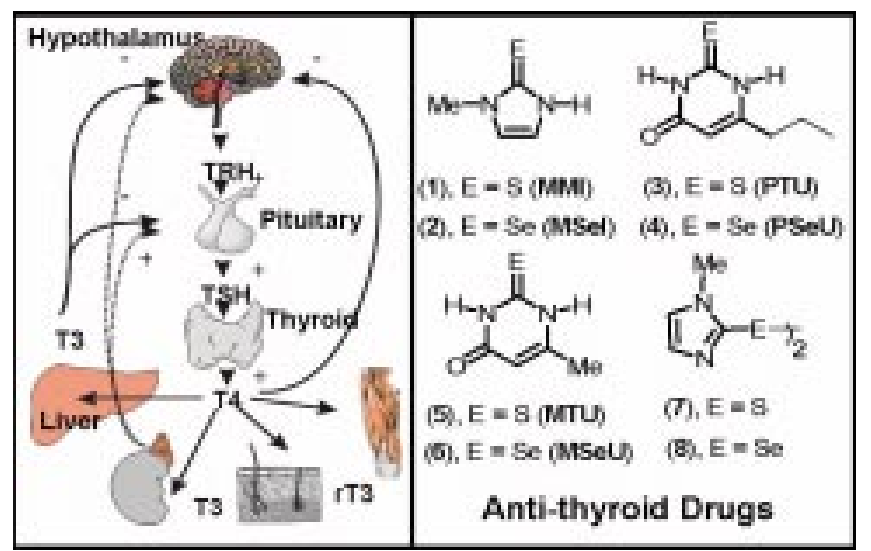

Figure 2. Pituitary feedback control system and antithyroid drugs. extended our approach to the synthesis and biological activities of a number of sulphur and selenium derivatives bearing the methimazole pharmacophore. ${ }^{6}$ In this paper, we discuss our experimental evidences that replacement of the sulphur atom in methimazole by selenium leads to a completely different mechanism. In addition, we describe the effect of a range of sulphur and selenium compounds bearing the methimazole moiety on peroxidase-catalysed oxidation reactions. We also describe the effect of substituents attached to the imidazole moiety on the selenolselone tautomerism by theoretical calculations.

\section{Results and discussion}

The tautomeric behaviour of MMI has been subjected to many investigations, ${ }^{7}$ which show that MMI exists almost exclusively as the thione tautomer (1a). Recent studies have shown that the thiourea-based drugs PTU and MTU also exist as thione tautomers. ${ }^{8}$ The stability of the thione tautomers may prevent these compounds from being oxidized to their corresponding disulphides, which may account for their high anti-thyroidal activity. Laurence et al have shown that the thione tautomer of MMI is responsible for its complexation with diiodine and the iodine complex of the thione tautomer $\mathbf{1 a}$ is favoured by $13.2 \mathrm{~kJ} \mathrm{~mol}^{-1}$ compared to that of the thiol tautomer $\mathbf{1 b}{ }^{9 \mathrm{a}}$ Therefore, the facile oxidation of $\mathbf{2}$ to the corresponding diselenide $(\mathbf{8})$ requires the compound to be in its zwitterionic form (2c) and not in the true selone form (2a). Although compound $\mathbf{2}$ can exist in both selenol and selone forms in solution, the ${ }^{77}$ Se NMR spectrum recorded immediately after the workup of the reaction showed a signal at $-5 \mathrm{ppm}$, which can be ascribed to the zwitterionic (2c) tautomer (figure 3). In the presence of air, the zwitterion slowly oxidizes to the corresponding diselenide and this process continues until all the zwitterion is converted to diselenide 8. In

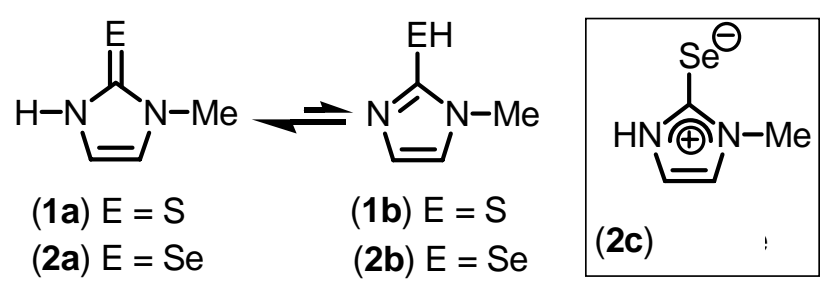

Figure 3. Proposed tautomeric structures of MMI and MSeI. 
contrast, the sulphur analogue, which exists predominantly in its thione tautomer form (1a), was found to very stable and could not beginning converted to the corresponding disulfide (7) even by using oxidizing agents such as $\mathrm{O}_{2}, \mathrm{H}_{2} \mathrm{O}_{2}$ etc. It should be mentioned that MMI is readily oxidized by the TPO system to form the disulfide. Although the oxidation of this compound by iodine has been postulated to be a possible mechanism, ${ }^{10}$ the chemical way through which MMI is transformed into disulfide $\mathbf{6}$ in vivo is unknown.

The theoretical investigations on selones are highly limited to the compounds having simple substituents, mainly due to the requirement of large basis sets for the calculations. ${ }^{11}$ The relatively larger size and greater polarizability of selenium as compared with sulphur have led to the assumption that the compounds with selone moiety are less stable than their sulphur analogues. Because the inhibition of TPO by antithyroid drugs depends upon the redox state of sulphur or selenium, we performed detailed quantum chemical calculations on $\mathbf{1}$ and $\mathbf{2}$ in gas phase. These studies show that the formation of the diselenide $(\mathbf{8})$ from $\mathbf{2 c}$ is energetically more favoured than the formation of the disulfide (7) from the corresponding thiol (1b). Interestingly, the conversion of thiol to thione is more favoured than the conversion of selenol to the corresponding selone. This can be rationalized by comparing the relative position of hydrogen on sulphur or selenium with respect to N1 in their most stable conformations. In the selenol (2b, figure 4), the $\mathrm{H}$ atom is located away from N1 leading to an increase in the energy barrier for the selenol-selone conversion. In contrast, the $\mathrm{H}$ atom is located in the close proximity of $\mathrm{N} 1$ in the thiol $(\mathbf{1 b}$, figure 2), which may favour the thiol-thione conversion. As the thione form is calculated to be much more stable than the corresponding thiol, it is quite unlikely that the thiol form contributes to the antithyroid activity of MMI. However, when the calculations were performed by including solvent effects, compound 2 was found to exist only in the zwitterionic form. Although the selone (2a) was also calculated to be more stable than the selenol $(\mathbf{2 b})$, the facile oxidation of the zwitterion to the corresponding diselenide disfavours the existence of selenol tautomer.

Although compound 2 readily oxidizes to diselenide $\mathbf{8}$, the corresponding zwitterion $(\mathbf{2 c})$ can be conveniently obtained by reducing the diselenide by $\mathrm{NaBH}_{4}$ or glutathione (GSH). The reaction of $\mathbf{8}$ with
$\mathrm{NaBH}_{4}$ followed by aqueous workup afforded the zwitterion as white solid, which was found to be stable under inert atmosphere and could be employed for in vitro biological assays without any noticeable oxidation. The treatment of diselenide 8 with 2 equiv. of GSH has also produced the zwitterion in nearly quantitative yield. Interestingly, the formation of a true selone (2a) was not observed in any of these processes, supporting the theoretical calculations that the selone exists as the zwitterion (2c) due to the nitrogen atoms present in the imidazole ring. The ${ }^{1} \mathrm{H}$ and ${ }^{13} \mathrm{C}$ NMR data and the large upfield shift in the ${ }^{77} \mathrm{Se}$ NMR chemical shift for the zwitterion $(-5 \mathrm{ppm})$, supports this assumption. In agreement with the theoretical data, the ${ }^{77} \mathrm{Se}$ NMR experiments show that the selone is more dissociated in water $(-53 \mathrm{ppm})$ than in organic solvent $(-5 \mathrm{ppm})$ as evidenced by a large upfield shift when changing the solvent from $\mathrm{CDCl}_{3}$ to $\mathrm{D}_{2} \mathrm{O}$. The facile reduction of 8 by GSH suggests that this compound may exist in its zwitterionic form under in vivo conditions, because GSH is present in the thyroid gland in high concentrations. It has been shown that GSH is an important antioxidant in thyroid gland and the peroxide scavenging activity of MMI is considerably increased by GSH. ${ }^{12}$

The enzyme inhibition experiments were carried out with Fe-containing lactoperoxidase (LPO) since it is readily available in purified form. Furthermore, LPO has been shown to behave very similarly to TPO with respect to iodination of thyroglobulin, the natural substrate, and other iodide acceptors. ${ }^{13}$ Edelhock et al have reported the inactivation of LPO by thiourea-based drugs using LPO-N-acetyltyrosylamide assay. ${ }^{14}$ We have employed 2,2'-azio-bis 3-ethylbenthiazoline-6-sulphonic acid (ABTS) and $\mathrm{H}_{2} \mathrm{O}_{2}$ as substrates to determine the half-maximal inhibitory concentration $\left(\mathrm{IC}_{50}\right)$ of test compounds. The $\mathrm{IC}_{50}$ values for the inhibition of LPO-catalysed oxidation of ABTS by 1-3 and $\mathbf{5}$ are summarized in table 2 .

To obtain reliable $\mathrm{IC}_{50}$ values for compound $\mathbf{2}$ and to make a direct comparison with the sulphur analogue, it is important to carry out the inhibition experiments with the completely reduced species. Therefore, we carried out the experiment with the reduced species (zwitterion, 2c), which was obtained by reducing the diselenide (8) with $\mathrm{NaBH}_{4}$ in an aqueous solution. As expected, MMI inhibited the LPO activity with an $\mathrm{IC}_{50}$ value of $7 \cdot 8 \mu \mathrm{M}$, which is much lower than that observed with PTU and MTU. The selenium analogue (2c) also inhibited LPO, and 


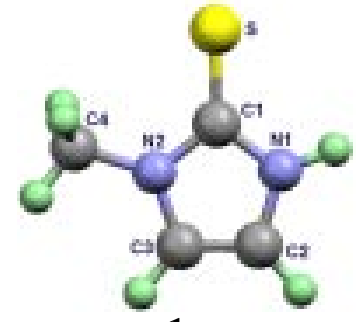

$1 \mathrm{a}$

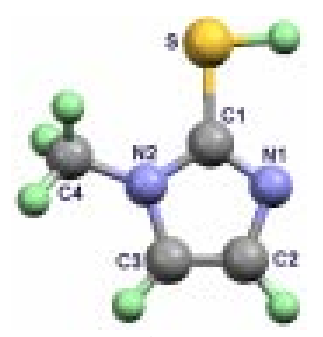

$1 \mathrm{~b}$

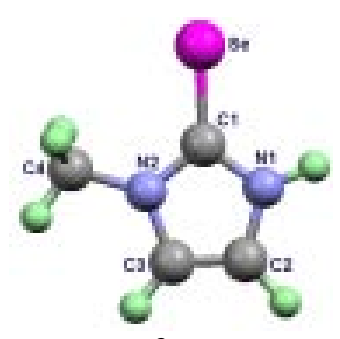

$2 \mathbf{a}$

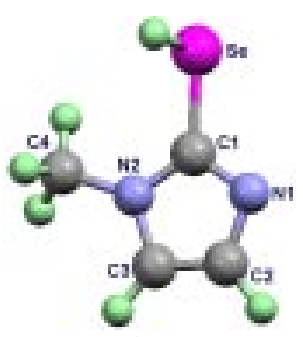

2b

Figure 4. Optimized geometries of $\mathbf{1}$ and $\mathbf{2}$. The conversion of thiol to thione is more favoured than the conversion of selenol to selone. The structures were optimised at the B3LYP level of theory using $6-311++\mathrm{G}(d, p)$ basis set.

Table 1. The theoretical data for $\mathbf{2}$ and $\mathbf{8}$ obtained by DFT calculations at B3LYP/6-311++G $(d, p)$ level along with the GIAO ${ }^{77} \mathrm{Se}$ NMR chemical shifts.

\begin{tabular}{lcc}
\hline Compound & $\begin{array}{c}\text { C-Se bond } \\
\text { length }(\AA)\end{array}$ & $\begin{array}{c}{ }^{77} \text { Se Chemical } \\
\text { shift }(\mathrm{ppm})^{\mathrm{b}}\end{array}$ \\
\hline $\mathbf{2 a}$ & 1.835 & 30 \\
$\mathbf{2 b}$ & 1.917 & -101 \\
$\mathbf{2 c}$ & 1.835 & 30 \\
$\mathbf{8}$ & $1.902^{\mathrm{a}}$ & 386 \\
\hline
\end{tabular}

${ }^{\mathrm{a} C}$ Calculated using B3LYP/6-31G(d) level; ${ }^{\mathrm{b}} \mathrm{NMR}$ values were calculated using B3LYP/6-311++G(2d,p) level and referenced to $\mathrm{Me}_{2} \mathrm{Se}$

Table 2. Inhibition of LPO activity by $\mathbf{1 - 3}$, and $\mathbf{5}$.

\begin{tabular}{lr}
\hline Compound & $\mathrm{IC}_{50}(\mu \mathrm{M})^{\mathrm{a}}$ \\
\hline MMI (1) & $7 \cdot 0 \pm 1 \cdot 1$ \\
MSeI (2) & $16 \cdot 4 \pm 1 \cdot 5$ \\
PTU (3) & $45 \cdot 0 \pm 2 \cdot 1$ \\
MTU (5) & $47 \cdot 8 \pm 0 \cdot 1$ \\
\hline
\end{tabular}

${ }^{\mathrm{a}}$ Concentration of the compound causing $50 \%$ inhibition. Each $\mathrm{IC}_{50}$ value was calculated from at least three independent experiments

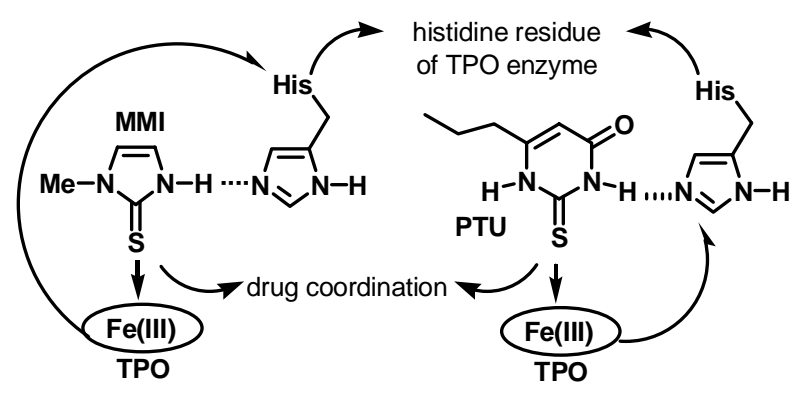

Figure 5. A hypothetical model for the coordination of thiourea drugs to the Fe-centre of TPO.

the $\mathrm{IC}_{50}$ value was found to be almost $4-5$ times lower than that of PTU and MTU. The higher activity of MMI as compared with PTU and MTU is in agreement with the previous studies on the inhibition of TPO. Since the activation of the iron centre in TPO must proceed through an interaction of $\mathrm{Fe}(\mathrm{III})$ with $\mathrm{H}_{2} \mathrm{O}_{2}$, TPO inactivation may occur through a competitive coordination of the drug to iron, assisted by hydrogen bonding with a histidine residue of the TPO enzyme (figure 5). Under these conditions, MMI might compete more successfully than PTU with $\mathrm{H}_{2} \mathrm{O}_{2}$, because the hydrogen-bond (hard) basicity $\mathrm{p} k_{\mathrm{HB}}$ value of MMI $(2 \cdot 11)$ is much higher than that of PTU ( 1.32). Similar to PTU, the methyl derivative $\mathbf{5}$ is also expected to be a weak inhibitor of TPO. On the other hand, compound $\mathbf{2}$, which exists predominantly in its zwitterionic form, does not have the ability to coordinate to the iron centre and, therefore, this compound must inhibit the LPO activity by a different mechanism.

Taurog et al have shown that MMI and related derivatives irreversibly inhibit LPO and TPO, leading to a complete inactivation of the enzymes. ${ }^{15}$ Doerge and others have shown that mammalian peroxidases including LPO may activate the anti-thyroid drugs through S-oxygenation to produce the corresponding sulphoxides or sulphenic acids. ${ }^{16}$ They have also shown that the irreversible inactivation of LPO and TPO by MMI proceeds through the S-oxygenation of the thione moiety to form a reactive sulphenic acid, which binds covalently to the prosthetic heme and irreversibly blocks enzyme activity. ${ }^{17}$ Given the higher reactivity of selenium compounds as compared with the sulphur derivatives towards oxidation, it is possible that the facile oxidation of the selenium compounds may lead to an efficient inhibition of LPO activity. With this in mind, we treated all the compounds in this study with $\mathrm{H}_{2} \mathrm{O}_{2}$ before adding LPO and ABTS. The LPO activity was measured several times by increasing the time for the reaction of the test compounds with $\mathrm{H}_{2} \mathrm{O}_{2}$. Remarkably, MSeI (2) inhibited the enzyme within few seconds 
even at lower concentrations, which can be ascribed to the facile oxidation of the reactive selenium moiety in 2 (MSeI) by $\mathrm{H}_{2} \mathrm{O}_{2}$. Because MMI also inhibits the enzyme very efficiently, we have carried out further experiments to prove that the mechanisms by which MMI and MSeI exert their inhibitory action are different. The initial rates $\left(v_{o}\right)$ derived from various concentrations of $\mathrm{H}_{2} \mathrm{O}_{2}$ were plotted against the concentration of $\mathrm{H}_{2} \mathrm{O}_{2}$. The LPO activity was completely inhibited by $40 \mu \mathrm{M}$ MMI and the enzyme's activity could not be recovered by increasing the $\mathrm{H}_{2} \mathrm{O}_{2}$ concentration (figure $6, \mathrm{~d}$ ). The LPO activity could not be recovered even at lower concentration of MMI $(10 \mu \mathrm{M})$ and higher concentration of $\mathrm{H}_{2} \mathrm{O}_{2}$ $(230 \mu \mathrm{M})$. This suggests that MMI does not act on $\mathrm{H}_{2} \mathrm{O}_{2}$, but it acts on the enzyme itself leading to an irreversible inhibition as previously proposed. On the other hand, 2 also inhibited the LPO activity as efficient as MMI, but in this case, the enzyme's activity could be completely recovered by increasing $\mathrm{H}_{2} \mathrm{O}_{2}$ concentration (figure $6, \mathrm{~b}$ ). The sigmoidal behaviour of the graph for this compound (figure $6, b$ ) is probably due to the utilization of $\mathrm{H}_{2} \mathrm{O}_{2}$ for the oxidation of selenenic acid to other oxidized products at lower concentration of the peroxide.

These observations strongly support the assumption that MSeI, in contrast to MMI, does not interfere with the enzyme directly, but it inhibits the LPO

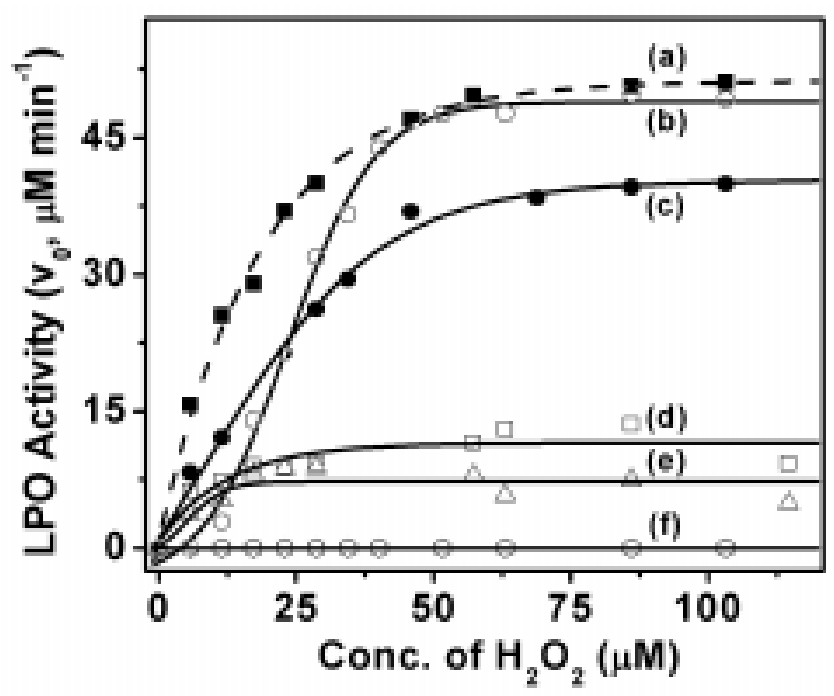

Figure 6. Plot of initial rates $\left(v_{o}\right)$ for the LPO-catalysed oxidation of ABTS vs concentration of $\mathrm{H}_{2} \mathrm{O}_{2}$. (a) Control activity, (b) $40 \mu \mathrm{M}$ of 2 , (c) $40 \mu \mathrm{M}$ of 8 , (d) $80 \mu \mathrm{M}$ of PTU, (e) $80 \mu \mathrm{M}$ of MTU, (f) $40 \mu \mathrm{M}$ of MMI. Conditions: LPO: $6.5 \mathrm{nM} ; \mathrm{H}_{2} \mathrm{O}_{2}: 22.9 \mu \mathrm{M}$. activity by reducing the $\mathrm{H}_{2} \mathrm{O}_{2}$, which is required for the oxidation of the iron centre in LPO (figure 7). When coupled with a suitable thiol such as GSH, compound 2 may constitute redox cycle involving a catalytic reduction of $\mathrm{H}_{2} \mathrm{O}_{2}$ (glutathione peroxidase (GPx) activity). ${ }^{18}$ In this way, compound 2 mimics the action of GPx, a selenoenzyme that protects the cellular components from oxidative damage by reducing $\mathrm{H}_{2} \mathrm{O}_{2}$ with the help of GSH. Recently, the GPx enzyme present in thyroid gland has been shown to inhibit the iodination reactions by degrading the intracellular $\mathrm{H}_{2} \mathrm{O}_{2} \cdot{ }^{19}$ In fact, the key compound 2 exhibited interesting GPx activity, leading to an assumption that some of the antithyroid drugs may act as antioxidants in addition to their inhibition behaviour. However, GSH does not appear to be a suitable thiol co-substrate for the catalytic antioxidant activity of $\mathbf{2}$, because the reaction of $\mathbf{2}$ with hydrogen peroxide was found to be much faster than the reduction of the intermediate selenenic acid and/or other oxidized species by GSH. Similar to the zwitterion-mediated inhibition, the inhibition by diselenide 8 could also be reversed by increasing the $\mathrm{H}_{2} \mathrm{O}_{2}$ concentration (figure 6, c). Although compound 8 did not give any new ${ }^{77} \mathrm{Se}$ NMR signal with one equiv. $\mathrm{H}_{2} \mathrm{O}_{2}$, addition of an excess amount of $\mathrm{H}_{2} \mathrm{O}_{2}$ to 8 produced a new signal at $1045 \mathrm{ppm}$, which cannot be ascribed to the selenenic acid because this signal appears to be different from that obtained from the reaction of 2 with $\mathrm{H}_{2} \mathrm{O}_{2}$, which showed a signal at $1207 \mathrm{ppm}$.

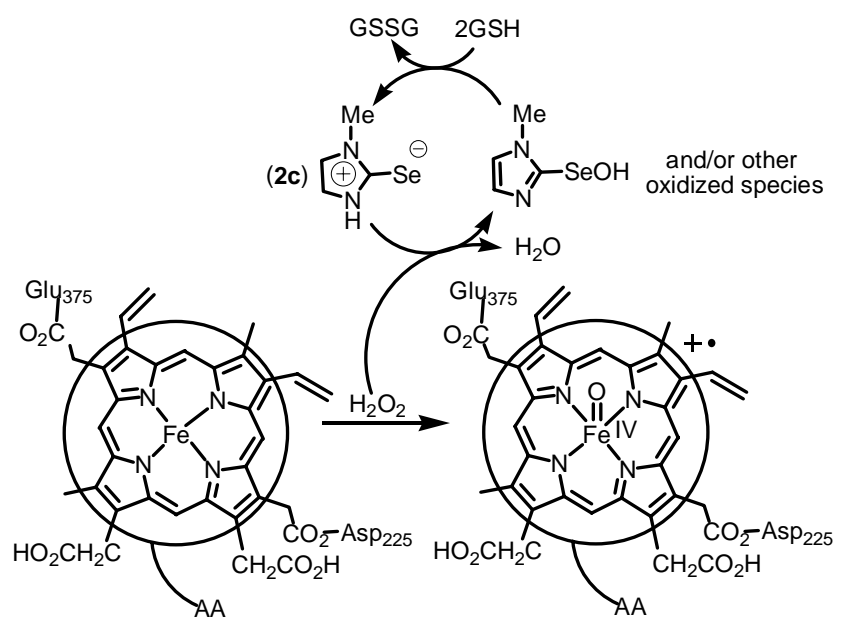

Figure 7. A hypothetical model representing the inhibition of LPO by 2 . The porphyrin core inside the circle represents the active centre of LPO. AA: amino acid residues. 
As expected, the plot of initial rates $\left(v_{o}\right)$ vs concentration of MSeI shows that the rate of the reaction decreases with increasing concentration of MSeI. In all these cases, the LPO activity could be recovered by increasing the hydrogen peroxide concentration. These experimental observations support the conclusions made by Taurog et al that MSeI, unlike MMI, cannot act as an irreversible inhibitor of TPO. These observations also support the in vivo experiments, which showed that MMI is at least 50 times more potent than MSeI as an inhibitor of organic iodine formation in the thyroid. Crucially, the treatment of 2 with the selenolate specific reagent, iodoacetic acid, abolished the inhibitory potency of $\mathbf{2}$, confirming that the oxidation of the selenium centre by $\mathrm{H}_{2} \mathrm{O}_{2}$ is responsible for the inhibition. In contrast, the sulphur analogue MMI was found to be less sensitive to the iodoacetic acid treatment and this also confirms that the thiol form of MMI is not only less predominant in solution, but also less reactive as compared with the thione form.

\section{Conclusion}

In summary, our experimental and theoretical studies show that the selenium analogue of methimazole (MSeI) exists predominantly in its zwitterionic form whereas the sulphur compound exists in its thione form and the oxidation of the zwitterion to the corresponding diselenide is energetically more favoured than the conversion of thione/thiol to the corresponding disulfide. Although MSeI readily oxidizes to produce the diselenide, the oxidized form can be easily reduced by reducing agents such as $\mathrm{NaBH}_{4}$ or glutathione (GSH). The ${ }^{77} \mathrm{Se}$ NMR studies show that the selone form of MSeI dissociates in solution to form a more reactive selenolate, which could be trapped by selenolate specific reagents such as iodoacetic acid. In its reduced form, MSeI effectively and reversibly inhibits the iron-containing lactoperoxidase (LPO). In contrast to methimazole, MSeI does not interfere with the enzyme directly, but it inhibits LPO by reducing the $\mathrm{H}_{2} \mathrm{O}_{2}$ that is required for the oxidation of the iron centre in LPO. In the presence of GSH, MSeI constitutes a redox cycle involving a catalytic reduction of $\mathrm{H}_{2} \mathrm{O}_{2}$ and thereby mimics the glutathione peroxidase (GPx) activity in vitro. These studies reveal that the degradation of the intracellular $\mathrm{H}_{2} \mathrm{O}_{2}$ by the selenium analogues of anti-thyroid drugs may be beneficial to the thyroid gland as these compounds may act as antioxidants and protect thyroid cells from oxidative damage. Because the drugs with an action essentially on $\mathrm{H}_{2} \mathrm{O}_{2}$ can reversibly inhibit the thyroid peroxidase, such drugs with a more controlled action could be of great importance in the treatment of hyperthyroidism.

\section{Acknowledgments}

This study was supported by the Department of Science and Technology (DST), and Council of Scientific and Industrial Research (CSIR), New Delhi. GM acknowledges the DST for the Ramanna Fellowship and GR thanks the CSIR for a research fellowship.

\section{References}

1. (a) Taurog A 1951 Thyroid hormone synthesis. In Werner's the Thyroid (eds) L E Braverman and R D Utiger, pp 51-97; (b) Taurog A, Doriss M L and Doerge D R 1996 Arch. Biochem. Biophys. 33024

2. (a) Berry M J, Banu L and Larsen P R 1991 Nature (London) 349 438; (b) Larsen P R and Berry M J 1995 Аnnu. Rev. Nutr. 15 323; (c) St Germain D L and Galton V A 1997 Thyroid 7 655; (d) Köhrle J 1999 Biochimie 81 527; (d) Bianco A C, Salvatore D, Gereben B, Berry M J and Larsen P R 2002 Endocr. Rev. 23 38; (e) Köhrle J 2002 Methods Enzymol. 347 125

3. (a) Buxeraud J, Absil A C, Claude J, Raby C, Catanzano G and Beck C 1985 Eur. J. Med. Chem. 20 43; (b) Raby C, Lagorce J F, Jambut-Absil A C, Buxeraud J and Catanzano G 1990 Endocrinology 126 1683

4. Bassosi R, Niccolai N and Rossi C 1978 Biophys. Chem. 861

5. (a) Visser T J, Kaptein E and Aboul-Enein H Y 1992 Biochem. Biophys. Res. Commun. 189 1362; (b) Taurog A, Dorris M L, Guziec L J and Guziec F S Jr 1994 Biochem. Pharmacol. 48 1447; (c) Guziec L J and Guziec F S Jr 1994 J. Org. Chem. 59 4691; (d) Taurog A, Dorris M L, Hu W-X and Guziec F S Jr 1995 Biochem. Pharmacol. 49701

6. (a) Roy G, Nethaji M and Mugesh G 2004 J. Am. Chem. Soc. 126 2712; (b) Roy G and Mugesh G 2005 J. Am. Chem. Soc. 127 15207; (c) Roy G, Nethaji M and Mugesh G 2006 Org. Biomol. Chem. 4 2883; (d) Roy G, Das D and Mugesh G 2007 Inorg. Chem. Acta 360 000, doi: 10.1016/j.ica.2006.07.052

7. Balestrero R S, Forkey D M and Russell J G 1986 Magn. Reson. Chem. 24651

8. Antoniadis C D, Corban G J, Hadjikakou S K, Hadjiliadis N, Kubicki M, Warner S and Butler I S 2003 Eur. J. Inorg. Chem. 81635

9. Laurence C, El Ghomari M J, Le Questel J-Y, Berthelot M and Mokhlisse R 1998 J. Chem. Soc., Perkin Trans. 21545 
10. Aragoni M C, Arca M, Demartin F, Devillanova F A, Garau A, Isaia F, Lippolis V and Verani G $2002 \mathrm{~J}$. Am. Chem. Soc. 1244538

11. (a) Ha T-K and Puebla C 1994 Chem. Phys. 181 47; (b) Jemmis E D, Giju K T and Leszczynski J 1997 J. Phys. Chem. A101 7389

12. Kim H, Lee T-H, Hwang Y S, Bang M A, Kim K H, Suh J M, Chung H K, Yu D-Y, Lee K-K, Kwon O-Y, Ro H K and Shong M 2001 Mol. Pharmacol. 60972

13. Taurog A, Dorris M L and Lamas L 1974 Endocrinology 941286

14. Edelhoch H, Irace G, Johnson M L, Michot J L and Nunez J 1979 J. Biol. Chem. 25411822
15. (a) Taurog A, Dorris M L and Guziec F S Jr 1989 Endocrinology 124 30, and references therein

16. (a) Doerge D R 1986 Arch. Biochem. Biophys. 244 678; (b) Kobayashi S, Nakano M, Goto T, Kimura T and Schaap A P 1986 Biochem. Biophys. Res. Commun. 135166

17. Doerge D R, Cooray N M and Brewster M E 1991 Biochemistry 308960

18. Sarma B K and Mugesh G 2005 J. Am. Chem. Soc. 12711477 , and references therein

19. (a) Björkman U, Ekholm R 1995 Mol. Cell. Endocrinol. 111 99; (b) Ekholm R and Björkman U 1997 Endocrinology 1382871 